\title{
RADIAL SYMMETRY OF THE FIRST EIGENFUNCTION FOR THE $p$-LAPLACIAN IN THE BALL
}

\author{
TILAK BHATTACHARYA
}

(Communicated by Walter Littman)

\begin{abstract}
We prove the radial symmetry of the eigenfunction corresponding to the first eigenvalue of the equation: $\operatorname{div}\left(|\nabla u|^{p-2} \nabla u\right)+\lambda|u|^{p-2} u=0$, when $\Omega$ is a ball in $R^{n}$ and $1<p<\infty$.
\end{abstract}

Introduction. Let $\Omega$ be a bounded domain in $R^{n}, n \geq 2$. Consider the problem

$$
\begin{gathered}
\Delta_{p} u+\lambda|u|^{p-2} u=0 \quad \text { in } \Omega, \\
u \in W_{0}^{1, p}(\Omega), u \not \equiv 0, \lambda \in R, \text { and } 1<p<\infty,
\end{gathered}
$$

where $\Delta_{p} u=\operatorname{div}\left(|\nabla u|^{p-2} \nabla u\right)$ is the $p$-Laplacian of $u$.

We say that $u$ is a solution of $\left(1_{\lambda}\right)$ if there exists a $\lambda$, and $\left(1_{\lambda}\right)$ holds in the sense of distributions, i.e.

$$
\int_{\Omega}|\nabla u|^{p-2} \nabla u \cdot \nabla \psi=\lambda \int_{\Omega}|u|^{p-2} u \psi \quad \forall \psi \in W_{0}^{1, p}(\Omega) .
$$

Consider the following minimization problem

$$
\inf I(v), \quad v \in W_{0}^{1, p}(\Omega), \quad J(v)=1,
$$

where $I(v)=(1 / p) \int_{\Omega}|\nabla v|^{p}$ and $J(v)=(1 / p) \int_{\Omega}|v|^{p-1} v$.

It can be shown $[1,2]$ that there exists a smallest $\lambda_{1}>0$ and $u_{\lambda_{1}}>0$ that solves $\left(1_{\lambda_{1}}\right)$. In that case $\lambda_{1}$ is the infimum in (2). Furthermore $u$ is a solution (2) iff $u$ solves $\left(1_{\lambda_{1}}\right)$ and satisfies $J(u)=1$. In the case $\Omega$ is a ball, the spherically decreasing rearrangements of positive solutions are also solutions. Furthermore if $\phi(r)$ is a radial solution then all other radial solutions are scalar multiples of $\phi(r)$ [1]. The radial solution $\phi(r)$ can be chosen to be positive.

In [1] the question is raised as to whether or not a solution, corresponding to $\lambda_{1}$ in a ball, must be radially symmetric. In the following we prove that if $u$ solves $\left(1_{\lambda_{1}}\right)$ in a ball, then $u$ is radially symmetric (see Theorem 1 ). Our method is based on an idea of Pólya and Szegö [4, p. 89].

Radial symmetry. Let $\Omega$ be the ball of radius $R$ centered at the origin. Let $u \in W_{0}^{1, p}(\Omega)$ solve $\left(1_{\lambda_{1}}\right)$. By a remark at the end of Theorem 4 in [5, p. 264], for $1<p \leq n,\|u\|_{\infty, \Omega} \leq C\|u\|_{p, \Omega}$. If $p>n$, by Theorem 7.17 in $\left[3\right.$, p. 163] $u \in C^{\beta}(\bar{\Omega})$ with $\beta=1-n / p$.

Received by the editors August 5, 1987.

1980 Mathematics Subject Classification (1985 Revision). Primary 35P30.

The author thanks Professors Allen Weitsman and Juan Manfredi for helpful discussions and encouragement. 
Thus $u \in L^{\infty}(\Omega)$ and by the results in $[7], u \in C_{\mathrm{loc}}^{1, \alpha}(\Omega)$. Let $\phi(r)>0$ be a radial solution of $\left(1_{\lambda_{1}}\right)$ and let $f$ be such that

$$
u=f \phi \text {. }
$$

By Theorem 3 in $[\mathbf{1}], \phi \in C^{1}(\bar{\Omega})$ and hence $f \in C^{1}(\Omega)$.

Let $\operatorname{vol}(S)$ denote the volume of a region $S$ in $R^{n}$ and $\lambda_{1}(S)$ denote the infimum of (2) in $S$.

We now prove a few preliminary results that lead to the main theorem. We begin with

PROPOSITION 1. Let $\Omega_{1}$ and $\Omega_{2}$ be balls of radius $R_{1}$ and $R_{2}$ centered at the origin with $R_{1}<R_{2}$. Then $\lambda_{1}\left(\Omega_{2}\right)<\lambda_{1}\left(\Omega_{1}\right)$.

Proof. Let $\phi_{1}$ be radial and minimize (2) in $\Omega_{1}$. We take $\phi_{1}>0$. Then $I\left(\phi_{1}\right)=\lambda_{1}\left(\Omega_{1}\right)$ and $J\left(\phi_{1}\right)=1$.

Set $\hat{\phi}(r)=c^{n / p} \phi_{1}(c r)$ where $c=R_{1} / R_{2}$. Then $\hat{\phi}\left(R_{2}\right)=0, J\left(\hat{\phi}_{2}\right)=1$, and $I\left(\hat{\phi}_{2}\right)=c^{p} \lambda_{1}\left(\Omega_{1}\right)$. But $\lambda_{1}\left(\Omega_{2}\right)$ is the infimum of $(2)$ in $\Omega_{2}$ and hence $\lambda_{1}\left(\Omega_{2}\right) \leq$ $c^{p} \lambda_{1}\left(\Omega_{1}\right)<\lambda_{1}\left(\Omega_{1}\right)$.

LEMMA 1. If $u \in W_{0}^{1, p}(\Omega)$ solves $\left(1_{\lambda_{1}}\right)$, then either $u>0$ or $u<0$ in $\Omega$.

PROOF. First we show that $u$ does not change sign. Suppose it does. Let $u^{+}=\max \{u, 0\}$ and $\Omega^{+}=\{x \in \Omega: u>0\}$. As $u^{+}$is continuous, $\Omega^{+}$is an open subset of $\Omega$ with $0<\operatorname{vol}\left(\Omega^{+}\right)<\operatorname{vol}(\Omega)$. Furthermore, $u^{+} \in W_{0}^{1, p}\left(\Omega^{+}\right)$. Scale $u^{+}$so that $J\left(u^{+}\right)=1$ in $\Omega^{+}$. Putting $u^{+}$as $\psi$ in $\left(1_{\lambda_{1}}^{\prime}\right)$ gives $I\left(u^{+}\right)=\lambda_{1}(\Omega) \geq \lambda_{1}\left(\Omega^{+}\right)$. Let $\Omega_{s}$ be the ball centered at the origin with $\operatorname{vol}\left(\Omega_{s}\right)=\operatorname{vol}\left(\Omega^{+}\right)$. By Theorem 2 in [1] (also see [6]), $\lambda_{1}\left(\Omega^{+}\right) \geq \lambda_{1}\left(\Omega_{s}\right)$. Proposition 1 then implies $\lambda_{1}(\Omega) \geq \lambda_{1}\left(\Omega^{+}\right) \geq$ $\lambda_{1}\left(\Omega_{s}\right)>\lambda_{1}(\Omega)$, a contradiction.

To prove that $u$ never vanishes in $\Omega$, we use the strong maximum principle as stated in Proposition 3.2.2 in [8].

Note. From now on we will take $u>0$. It then follows from (3) that $f>0$ in $\Omega$.

For notational simplicity we write

$$
\|u\|_{p}=\|u\|_{p, \Omega}, \quad 1 \leq p \leq \infty .
$$

LEMMA 2. If $u \in W_{0}^{1, p}(\Omega)$ solves $\left(1_{\lambda_{1}}\right)$ and $\phi(r)>0$ is a radial solution of $\left(1_{\lambda_{1}}\right)$, and if $f$ is such that $u=f \phi$, then $f \in L^{\infty}(\Omega)$.

PROOF. Now $f=u / \phi$ where $\phi(r)$ is never zero. Let $\Omega_{1}$ be the ball of radius $R_{1}$ centered at the origin with $0<R_{1}<R$. Then

$$
\sup _{\Omega_{1}} f \leq\|u\|_{\infty} / \inf _{\Omega_{1}} \phi \leq M<+\infty .
$$
Then

Consider the annulus $A=\Omega \backslash \bar{\Omega}_{1}$. Define $w(r)=k(R-r)$ for $k>0$ where $r=|x|$.

$$
\Delta_{p} w=-(n-1) k^{p-1} / r .
$$

Choose $k$ so large such that

$$
-(n-1) k^{p-1} / R<-\lambda_{1}\|u\|_{\infty}^{p-1} \text { and } \quad k\left(R-R_{1}\right)>\|u\|_{\infty} .
$$

Then $\Delta_{p} w \leq \Delta_{p} u$ with $w \geq u$ on $\partial A$. By the weak comparison principle, $w \geq u$ in $A$. Thus $u(x) \leq k(R-|x|)$ with $k$ as chosen in (4). By Theorem 3 in [1] and 
Proposition 3.2.1 in [8], there is a $c>0$ such that $\phi^{\prime}(r) \leq-c<0$ in $R_{1} \leq r \leq R$. Hence, $\phi(r) \geq c(R-r)$ in $A$. This implies $f=u / \phi \leq k / c<+\infty$ in $A$. Thus $f \in L^{\infty}(\Omega)$.

LEMMA 3. We have $f^{p} \phi \in W_{0}^{1, p}(\Omega)$.

ProOF. It is obvious that $f^{p} \phi \in L^{p}(\Omega)$. Now

$$
\nabla\left(f^{p} \phi\right)=f^{p} \nabla \phi+p \phi f^{p-1} \nabla f .
$$

Also

$$
\nabla f=\frac{\nabla u}{\phi}-\frac{u}{\phi^{2}} \nabla \phi=\frac{\nabla u}{\phi}-f \frac{\nabla \phi}{\phi} .
$$

Hence

$$
\phi \nabla f=\nabla u-f \nabla \phi
$$

The above yields

$$
\left|\nabla\left(f^{p} \phi\right)\right| \leq f^{p}|\nabla \phi|+p f^{p-1}\{|\nabla u|+f|\nabla \phi|\} .
$$

As $f, \phi, \nabla \phi \in L^{\infty}(\Omega)$ and $u, \phi \in W_{0}^{1, p}(\Omega)$, it follows that $f^{p} \phi \in W^{1, p}(\Omega)$. We now show $f^{p} \phi$ is the strong limit in $W^{1, p}(\Omega)$ of a sequence of functions belonging to $C_{0}^{1}(\Omega)$.

For $n=2,3,4, \ldots$, let $R_{n}=(n-1) R / n$ and $\bar{R}_{n}=R_{n}+R / 2 n$, and $\Omega_{n}$ be the ball of radius $R_{n}$ centered at the origin. For each $n$, let $0 \leq h_{n}(r) \leq 1$ be a radial function in $C_{0}^{1}(\Omega)$ such that

$$
h_{n}(r)= \begin{cases}1 & \text { in } 0 \leq r \leq R_{n} \\ 0 & \text { in } \bar{R}_{n} \leq r \leq R\end{cases}
$$

and $\left|\nabla h_{n}\right| \leq 8 n / R$. Then the functions $g_{n}=\left(f^{p} \phi\right) h_{n}$ belong to $C_{0}^{1}(\Omega)$. We will show that the functions $g_{n}$ converge to $f^{p} \phi$ in $W^{1, p}(\Omega)$.

By Theorem 3 in $[\mathbf{1}], \phi \in C^{1}(\bar{\Omega})$ and $\phi$ vanishes on $\partial \Omega$. Thus there exists a $c>0$ such that $\phi^{\prime}(r) \geq-c$ in $R / 2 \leq r \leq R$. Integrating, we obtain that $\phi(r) \leq c(R-r)$ in $R / 2 \leq r \leq R$. In particular, it follows that $\phi(r) \leq c\left(R-R_{n}\right)$ in $R_{n} \leq r \leq \bar{R}_{n}$. This implies that

$$
\phi(r) \leq c R / n \quad \text { in } R_{n} \leq r \leq \bar{R}_{n} .
$$

We first show that $g_{n} \rightarrow f^{p} \phi$ in $L^{p}(\Omega)$ as $n \rightarrow \infty$. We have

$$
\begin{aligned}
\int_{\Omega}\left|g_{n}-f^{p} \phi\right|^{p} & =\int_{\Omega}\left(f^{p} \phi\right)^{p}\left|1-h_{n}\right|^{p} \leq\left\|f^{p} \phi\right\|_{\infty}^{p} \int_{\Omega \backslash \Omega_{n}} 1 \\
& \leq\left\|f^{p} \phi\right\|_{\infty}^{p} \operatorname{vol}\left(\Omega \backslash \Omega_{n}\right) .
\end{aligned}
$$

The result is obtained by noting that $\operatorname{vol}\left(\Omega \backslash \Omega_{n}\right) \rightarrow 0$ as $n \rightarrow \infty$. Next we show that $\nabla g_{n} \rightarrow \nabla\left(f^{p} \phi\right)$ in $L^{p}(\Omega)$. By Minkowski's inequality

$$
\begin{gathered}
\left(\int_{\Omega}\left|\nabla g_{n}-\nabla\left(f^{p} \phi\right)\right|^{p}\right)^{1 / p} \leq\left(\int_{\Omega}\left|\nabla\left(f^{p} \phi\right)\right|^{p}\left|1-h_{n}\right|^{p}\right)^{1 / p}+\left(\int_{\Omega}\left(f^{p} \phi\right)^{p}\left|\nabla h_{n}\right|^{p}\right)^{1 / p} \\
\leq\left(\int_{\Omega \backslash \Omega_{n}}\left|\nabla\left(f^{p} \phi\right)\right|^{p}\right)^{1 / p}+\left\|f^{p}\right\|_{\infty}\left(\int_{\Omega \backslash \Omega_{n}} \phi^{p}\left|\nabla h_{n}\right|^{p}\right)^{1 / p} .
\end{gathered}
$$


The first term on the right side of the inequality can be made small by the absolute continuity of the integral. We estimate the second term as follows. Using (6), we obtain that

$$
\phi^{p}\left|\nabla h_{n}\right|^{p} \leq(c R / n)^{p}(8 n / R)^{p} \leq M<+\infty \quad \forall n=2,3,4, \ldots
$$

Hence

$$
\int_{\Omega \backslash \Omega_{n}} \phi^{p}\left|\nabla h_{n}\right|^{p} \leq M \operatorname{vol}\left(\Omega \backslash \Omega_{n}\right) .
$$

Since $\operatorname{vol}\left(\Omega \backslash \Omega_{n}\right) \rightarrow 0$ as $n \rightarrow \infty$, we obtain that $\nabla g_{n} \rightarrow \nabla\left(f^{p} \phi\right)$ in $L^{p}(\Omega)$. This completes the proof.

LEMMA 4. The following integrals exist:

(i) $I_{1}=\int_{\Omega} f^{p}|\nabla \phi|^{p}<\infty$,

(ii) $I_{2}=\left.\int_{\Omega}\left|f^{p-2} \phi(\nabla \phi \cdot \nabla f)\right| \nabla \phi\right|^{p-2} \mid<\infty$,

(iii) $I_{3}=\int_{\Omega} \phi^{p}|\nabla f|^{p}<\infty$.

ProOF. We note using (5) that

$$
|\phi(\nabla \phi \cdot \nabla f)| \leq|\nabla \phi|\{|\nabla u|+f|\nabla \phi|\}
$$

and

$$
\phi^{2}|\nabla f|^{p} \leq(|\nabla u|+f|\nabla \phi|)^{p} .
$$

Thus $I_{1}, I_{2}, I_{3}$ exist, as $f, \phi, \nabla \phi \in L^{\infty}(\Omega)$ and $u, \phi \in W_{0}^{1, p}(\Omega)$.

Proposition 2. Let $A$ and $B$ be vectors in $R^{n}, n \geq 2$. If $1<p<\infty$, then

$$
|A+B|^{p} \geq|A|^{p}+p|A|^{p-2} A \cdot B+K|B|^{p}
$$

where $|V|$ denotes the length of a vector $V$, and

$$
K= \begin{cases}\min \left(1, \frac{|A+B|^{p}-|A|^{p}-p|A|^{p-2} A \cdot B}{|B|^{p}}\right) ; & B \neq 0, \\ 0, & B=0 .\end{cases}
$$

Furthermore, $0 \leq K \leq 1$ and $K=0$ iff $B=0$.

Before proving Proposition 2, we prove the following Lemma.

LEMMA 5. If $t \geq 0, \alpha$, and $s$ are any real numbers, and if $1<p<\infty$, then

$$
\left\{(1+\alpha)^{2} t^{2}+s^{2}\right\}^{p / 2} \geq(1+\alpha p) t^{p} .
$$

Equality holds in (8) iff $s=0$ and either $\alpha=0$ or $t=0$.

PrOOF. Observe that for all $s$,

$$
\left((1+\alpha)^{2} t^{2}+s^{2}\right)^{p / 2} \geq|1+\alpha|^{p} t^{p}
$$

To prove (8), it is then sufficient to prove that for all $\alpha$

$$
|1+\alpha|^{p} \geq 1+\alpha p
$$

Case 1. If $1+\alpha \geq 0$, then $|1+\alpha|^{p}=(1+\alpha)^{p}$. Define

$$
F(\alpha)=(1+\alpha)^{p}-1-\alpha p \quad \forall \alpha \geq-1 .
$$

Then

$$
F^{\prime}(\alpha)=p(1+\alpha)^{p-1}-p
$$


It is easy to see that $F^{\prime}(\alpha)<0$ when $-1 \leq \alpha<0$, and $F^{\prime}(\alpha)>0$ when $\alpha>0$. Noting that $F(0)=0$, we obtain (10). It is clear that, in the case $1+\alpha \geq 0$, equality in (10) holds, iff $\alpha=0$.

Case 2. If $1+\alpha<0$, then $1+\alpha p<0$ so (10) follows trivially, and strict inequality holds.

To prove the 'only if' part of the last assertion of the lemma, we note that for equality in (8), we must have equality in (9). This implies that $s=0$. If $t \neq 0$, then equality must hold in (10), which implies that $\alpha=0$.

ProOf OF Proposition 2. If $A=0$, the inequality in (7) follows easily. Let us assume that $A \neq 0$. Then we can write $B=\alpha A+D$, for some scalar $\alpha$ and some vector $D$ orthogonal to $A$. Such a decomposition is unique. Then

$$
|A+B|^{p}=\left((1+\alpha)^{2}|A|^{2}+|D|^{2}\right)^{p / 2}
$$

and

$$
|A|^{p}+p|A|^{p-2} A \cdot B=(1+\alpha p)|A|^{p}
$$

From Lemma 5

$$
|A+B|^{p} \geq|A|^{p}+p|A|^{p-2} A \cdot B .
$$

where equality holds iff $B=0$. Thus, $K$ in (7) is strictly positive whenever $B \neq 0$. This finishes the proof.

We now prove the main theorem of this paper.

THEOREM 1. Let $\Omega$ be a ball in $R^{n}$ and $u \in W_{0}^{1, p}(\Omega)$ solve $\left(1_{\lambda_{1}}\right)$. Then $u$ is radially symmetric.

ProOF. By the note at the end of Lemma $1, u>0$, and hence (2) yields

$$
\int_{\Omega}|\nabla u|^{p}=\lambda_{1} \int_{\Omega} u^{p}
$$

Using (3), we get

$$
\int_{\Omega}|\nabla(f \phi)|^{p}=\lambda_{1} \int_{\Omega}(f \phi)^{p}
$$

By Lemma $3, f^{p} \phi$ is a legitimate test function in $\left(1_{\lambda_{1}}^{\prime}\right)$. Using $\psi=f^{p} \phi$, and replacing $u$ by $\phi,\left(1_{\lambda_{1}}^{\prime}\right)$ gives

$$
\int_{\Omega}|\nabla \phi|^{p-2} \nabla \phi \cdot \nabla\left(f^{p} \phi\right)=\lambda_{1} \int_{\Omega}(f \phi)^{p}
$$

Hence,

$$
\int_{\Omega}|\nabla(f \phi)|^{p}=\int_{\Omega}|\nabla \phi|^{p-2} \nabla \phi \cdot \nabla\left(f^{p} \phi\right) .
$$

Now,

$$
\int_{\Omega}|\nabla(f \phi)|^{p}=\int_{\Omega}|f \nabla \phi+\phi \nabla f|^{p}
$$

Setting $A=f \nabla \phi$ and $B=\phi \nabla F$, and using (7), we have

$$
\int_{\Omega}|\nabla(f \phi)|^{p} \geq \int_{\Omega} f^{p}|\nabla \phi|^{p}+p f^{p-1} \phi|\nabla \phi|^{p-2}(\nabla \phi \cdot \nabla f)+K \phi^{p}|\nabla f|^{p},
$$


where $K$ is as defined in Proposition 2. Combining the first two terms on the right side of the inequality we obtain

$$
\int_{\Omega}|\nabla(f \phi)|^{p} \geq \int_{\Omega}|\nabla \phi|^{p-2} \nabla \phi \cdot \nabla\left(f^{p} \phi\right)+K \phi^{p}|\nabla f|^{p} .
$$

Comparing (11) and (12), we get

$$
\int_{\Omega} K \phi^{p}|\nabla f|^{p} \leq 0
$$

As $\phi>0$ in $\Omega$, we obtain $K|\nabla f|^{p}=0$. From Proposition $2, K$ and $\nabla f$ vanish together. Hence $f$ is a constant and $u$ is radially symmetric.

Note added in proof. The author has been able to extend this method to prove that $\lambda_{i}$ is simple for $C^{2}$ domains. The details are worked out in his Ph.D. thesis supervised by A. Weitsman. He has also learned that S. Sakaguchi and A. Anane have obtained similar results.

\section{REFERENCES}

1. F. De Thelin, Sur l'espace propre associè à la première valeur propre du pseudo-laplacien, Comptes Rendus 303 (1986), 355-358.

2. $\_$Q Quelques résultats d'existence et de non-existence pour une E.D.P. elliptique non linéaire, Comptes Rendus 299 (1984), 911-914.

3. D. Gilbarg and N. S. Trudinger, Elliptic partial differential equations of second order, SpringerVerlag, Berlin and New York, 1983.

4. G. Pólya and G. Szegö, Isoperimetric inequalities in mathematical physics, Ann. of Math. Studies, no. 27, Princeton Univ. Press, Princeton, N.J., 1951.

5. J. Serrin, Local behaviour of solutions of quasilinear equations, Acta Math. 111 (1964), 247-302.

6. E. Sperner, Jr., Symmetrisierung für Funktionen mehrerer reeler Variablen, Manuscripta Math. 11 (1974), 159-170.

7. P. Tolksdorf, Regularity for more general class of quasilinear elliptic equations, J. Differential Equations 51 (1984), 126-150.

8. Comm. Partial Differential Equations 8 (1983), 773-817.

Department of Mathematics, Purdue University, West Lafayette, Indiana 47907 60201

Current address: Department of Mathematics, Northwestern University, Evanston, Illinois 